\title{
Restrictions on the parameters of the Minimal Supersym- metric Standard Model with CP-violation
}

\author{
Albina Gurskaya ${ }^{1, *}$ and Mikhail Dolgopolov ${ }^{1, * *}$ and Elza Rykova $^{1, * * *}$ \\ ${ }^{1}$ Samara University, Academician Pavlov 1, 443011 Samara, Russia
}

\begin{abstract}
The minimal supersymmetric extension of the standard model with CP-violation is considered in this paper. The main area of the model parameters, which is attractive for researchers, corresponds to small values of the tangent mixing angle of two doublets Higgs fields $(\tan \beta)$. The authors discuss the possibilities of the model in correlation with the data on the search for the lightest stable supersymmetric particle. The masses of neutral Higgs bosons in the basis of CP-violationwas calculated.The results are presented as dependencies neutral CP-odd Higgs boson mass $m_{A}$ on $\tan \beta$. At small values $\operatorname{tg} \beta$ the mass of the lightest stable particle must be more than $\sim 250 \mathrm{GeV}$.
\end{abstract}

\section{Introduction}

Supersymmetry attracts many researchers, because it allows to solve some difficulties of the standard model, for example, the hierarchy of masses of fundamental fermions, the union of fundamental constants on a certain energy scale and others. Also new particles of the theory can be considered as candidates for the role of dark matter particles. The possibilities of supersymmetric models do not end here.

Minimum supersymmetric standard model (MSSM) is already considering on Large Hadron Collider (LHC) nowadays using specific scenarios, so called, benchmark scenario [1-4]. A characteristic feature of these scenarios is that the authors leave only two free parameters. This is the mass of the light CP-odd neutral Higgs boson $m_{A}$ and the tangent of the mixing angle of the two doublets of Higgs fields $\tan \beta$. But almost all of these scenarios have been closed by the accelerator experiment nowadays.

One of the directions of the search for supersymmetry is the search for the lightest stable superparticle, which, at the same time, is a candidate for the role of a dark matter particle (neutralino $\tilde{\chi}^{0}$ ) [5]. Several processes have been selected to search for this particle. For example, there are restrictions on processes such as $\tilde{g} \rightarrow q \tilde{q} \tilde{\chi}_{1}^{0}, \tilde{q} \rightarrow q \tilde{\chi}_{1}^{0}, \tilde{g} \rightarrow b \tilde{b} \tilde{\chi}_{1}^{0}, \tilde{q}_{L} \rightarrow$ $q W \tilde{\chi}_{1}^{0}, \tilde{g} \rightarrow t \tilde{\chi} \tilde{\chi}_{1}^{0}$. The neutralino mass must be more than $1 \mathrm{TeV}$ at mass gluino more than about 1.6 TeV according experimental data. Of course, its have their own limitations for each individual process. We give a lower value here, i.e. there are more serious restrictions. And in such processes as $\tilde{q}_{L} \rightarrow q W Z \tilde{\chi}_{1}^{0}, \tilde{g} \rightarrow q \tilde{q} W \tilde{\chi}_{1}^{0}, \tilde{q}, \tilde{b}, \tilde{t} \rightarrow q(\gamma / Z) \tilde{G}$ via $\tilde{\chi}_{1}^{0}, \tilde{g} \rightarrow q \tilde{q} W Z \tilde{\chi}_{1}^{0}$ there are weaker restrictions: $m_{\tilde{\chi}_{1}^{0}}>500 \mathrm{GeV}$ at $m_{\tilde{q}}>0.6-1.8 \mathrm{TeV}$.

\footnotetext{
*e-mail: a-gurska@yandex.ru

**e-mail: mvdolg@yandex.ru

***e-mail: elzarykova@rambler.ru
} 
In any case, the closure of benchmark scenarios does not mean that supersymmetry does not exist. In principle, all possible parameter regions are difficult to test in full on the experiment. Another possibility for "saving" the model lies in its modification. One motivation for this may be to try to explain the baryon asymmetry. This problem is directly related to the inclusion of new sources of CP-violation in the model. In particular, the researchers are looking at CP-violation in the Higgs sector. The authors considered the MSSM with CP-violation earlier $[6,7]$.

In this paper there are some estimates on the mass of the neutral Higgs boson and the neutralino mass for the MSSM which was developed by the authors using modern experimental data the LHC.

\section{Investigation of MSSM free parameters}

A description of the MSSM can be found in the works [6, 7]. Inclusion of CP-invariance violation is carried out in the effective potential method. The effective Higgs potential may include members that explicit violate CP-symmetry, as well as additional phases of spontaneous CP-violation in vaccum. In the simplest case, the effective potential also contains parameters that on the energy scale of the standard model are nonzero and that they violate the $\mathrm{CP}$-invariance explicitly. But these non-zero values are not explained. We believe that these parameters have contributions from supersymmetric partners of heavy fermions (squarks). In addition, we require vacuum stability and find the masses of neutral Higgs bosons at the local minimum of potential.

The transition to CP-mixed states is also realized by an additional rotation from a basis with CP-defined neutral Higgs bosons $(h, H, A)$ to a basis with CP-violation $\left(h_{1}, h_{2}, h_{3}\right)$ using an additional rotation matrix $\mathrm{A}$ :

$$
\left(\begin{array}{c}
h \\
H \\
A
\end{array}\right)=A\left(\begin{array}{l}
h_{1} \\
h_{2} \\
h_{3}
\end{array}\right)
$$

Hence, the given turn at diagonalization of the mass matrix of neutral Higgs bosons $m$ : $\left(h_{i}\right)^{T}\left(A^{-1}\right)^{T} m A^{-1} h_{i}$ give us the squares of the three neutral Higgs bosons masses:

$$
\mathcal{M}_{D}=\left(\begin{array}{ccc}
M_{h_{1}}^{2} & 0 & 0 \\
0 & M_{h_{2}}^{2} & 0 \\
0 & 0 & M_{h_{3}}^{2}
\end{array}\right),
$$

We consider further the dependences (fig. 1) of the neutral CP-odd Higgs boson on the tangent of the mixing angle of two Higgs doublets as in benchmark scenarios in the range $m_{A}=100 \div 500 \mathrm{GeV}$ and $\tan \beta=3 \div 50$. The remaining parameters are fixed: $M_{\text {susy }}=500$ $\mathrm{GeV}, \mu=500 \mathrm{GeV}, A_{t}=A_{b}=1000 \mathrm{GeV}$. The mass of the neutral CP-odd Higgs boson at large $\tan \beta$ is almost independent on the angle. We can see the dependence on small values of $\tan \beta$. The masses of CP-odd Higgs bosons are predominantly in the region greater than 1 $\mathrm{TeV}$.

We focused our attention on the area in the small angles and considered them separately, and did it so that limited $m_{h_{1}} \leq 127 \mathrm{GeV}$. The new parameter areas are represented by figures 2. The white area is the area the heavy neutral Higgs bosons with undefined CP-parity. This area was excluded from consideration. Blue area on the contour plot corresponds small values of neutral Higgs boson with lightest mass on figure $2 \mathrm{a}$. In this area, there are many opportunities to implement a model on an experiment as for heavy $m_{A}$ and as for light $m_{h_{1}}$. 


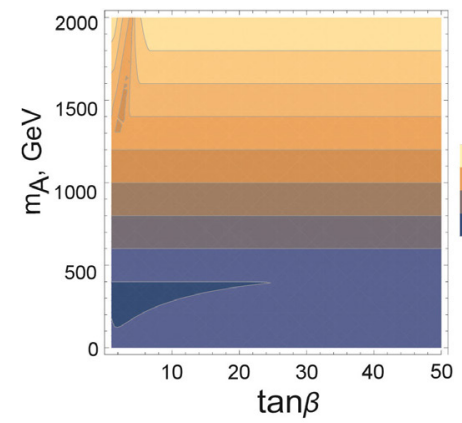

a)

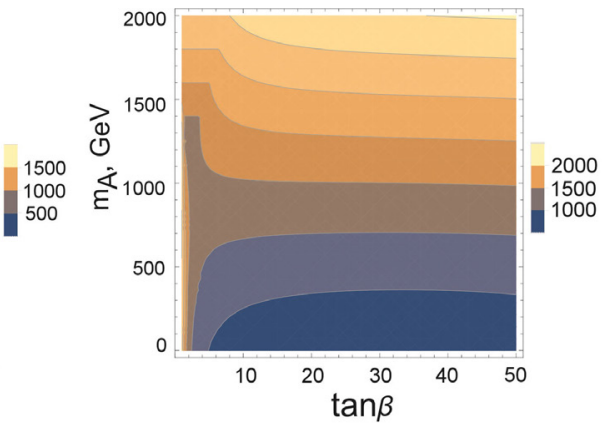

b)

Figure 1. Dependences $m_{A}$ on $\tan \beta$ : a) for $m_{h_{1}}$, b) for $m_{h_{2}}$.

The last possibility is probably talking about non-observed a light Higgs boson. The possibility of the existence of a light Higgs boson was discussed by the authors in a more General two-doublet model [8].

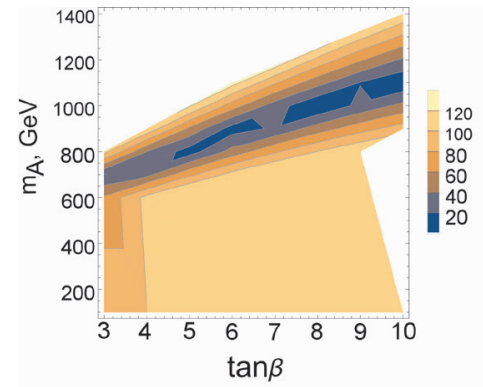

a)

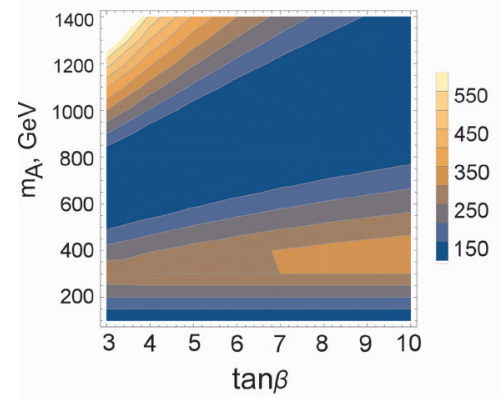

c)

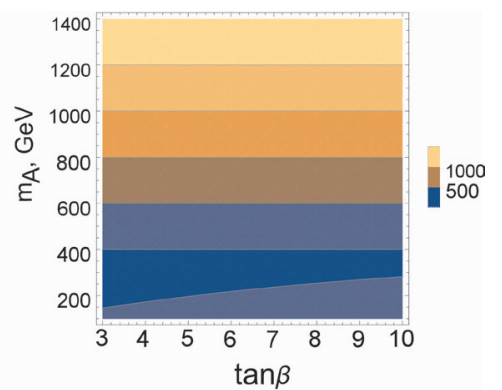

b)

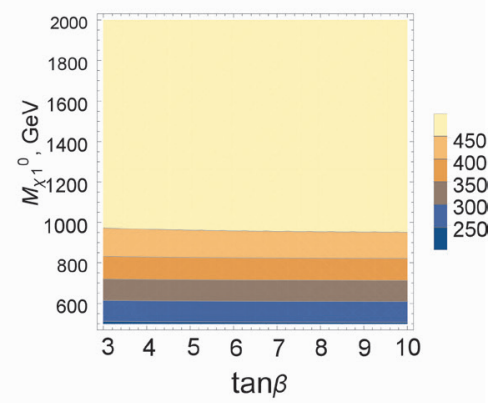

d)

Figure 2. Dependences $m_{A}$ on $\tan \beta$ : a) for $m_{h_{1}}$, b) for $m_{h_{2}}$, c) for $m_{h_{3}}$, d) for $m_{\tilde{\chi}_{1}^{0}}$. The white area is the area the heavy neutral Higgs bosons with undefined CP-parity. This area was excluded from consideration.

The comparison of masses dependencies $m_{h_{2}}$ and $m_{h_{3}}$ give us interesting result. There are areas when $m_{h_{3}}<m_{h_{2}}$ with light $m_{h_{1}}$ at the same time. We also calculated the neutralino mass for the selected data for the free parameters of the model. At small values $\operatorname{tg} \beta$ the mass of the lightest stable particle must be more than $\sim 250 \mathrm{GeV}$ (fig. $2 \mathrm{~d}$ ). 


\section{Summary}

We considered the masses of neutral CP-undefined neutral Higgs bosons in the MSSM. Also we can conclude that the masses of Higgs bosons at small $\operatorname{tg} \beta$ values have a complex dependence on the model parameters. There is a possibility of an unobservable light Higgs boson. At small values $\operatorname{tg} \beta$ the mass of the lightest stable particle must be more than $\sim 250 \mathrm{GeV}$.

Despite closed CP-preserving MSSM scenarios, there is many opportunities for searching for heavy supersymmetry within the MSSM. CP-invariance violation is also considered in the framework of more extended (non-minimal) models and give us rich phenomenology.

Acknowledgements. Authors are grateful to organizers of The XXIV International Workshop High Energy Physics and Quantum Field Theory for the attention and opportunities of discussing results of investigations.

\section{References}

[1] M. Carena at el. Eur.Phys.J.C 26, 601-607 (2003)

[2] M.Carena at el. Eur.Phys.J.C 73, 2552 (2013)

[3] M.Carena at el. Phys.Lett.B 495, 155-163 (2000)

[4] M.Carena at el. JHEP 02, 123 (2016)

[5] https://atlas.web.cern.ch/Atlas/GROUPS/PHYSICS/PUBNOTES/ATL-PHYS-PUB2019-022

[6] Akhmetzyanova E.N., Dolgopolov M.V., Dubinin M.N. Physics of Particles and Nuclei 36, S41-S44 (2005)

[7] Akhmetzyanova E.N. et al. Vestnik SamGU. Estestvennonauchnaya seriya 2(28), 122136 (2003)

[8] Akhmetzyanova E.N., Dolgopolov M.V., Smirnov I.A., Dubinin M.N. Physics of Atomic Nuclei 68, 541-548 (2005) 